\title{
LA DISTRIBUCIÓN DE LAS ZIGENAS (LEPIDOPTERA, ZYGAENIDAE) IBÉRICAS: UNA CONSECUENCIA DEL EFECTO PENÍNSULA
}

\author{
J. Martín Cano* y P. Gurrea*
}

\begin{abstract}
RESUMEN
Se ha analizado la distribución de las especies del género Zygaena presentes en la Península Ibérica. El método empleado ha sido el análisis de la densidad de especies en cuadrículas de igual superficie. Como variables independientes se han utilizado factores geográficos, físicos y climáticos. Los más relevantes para la densidad de especies de zigenas son la longitud geográfica y la distancia a Pirineos. En segundo lugar está la altitud, y finalmente otros factores como el tipo de suelo y la irregularidad pluviométrica. El modelo resultante es muy parecido al que presentan las mariposas ibéricas, y al igual que en éstas, puede asignarse a un marcado efecto península. El análisis de parsimonia pone de manifiesto la importancia de la parte oriental de la Península Ibérica como corredor biogeográfico para estas especies.
\end{abstract}

Palabras clave: Zygaena, efecto península, latitud, longitud, distancia a Pirineos, "mediterraneidad", Península Ibérica.

\section{ABSTRACT \\ The distribution of the Iberian burnets (Lepidoptera, Zygaenidae): a consequence of the peninsular effect}

The distribution of the burnets of the genus Zygaena in the Iberian Peninsula has been analysed. The method used has been the comparison of species densities in grids of equal area. Geographical, physical and climatic factors have been used as independent variables. The most relevant variables for the burnet species densities are the geographical longitude and the distance to the Pyrenees. The second most important factor is altitude and finally other variables as soil type and climatic irregularity. The resulting model is very similar to that of the Iberian butterflies, and, similarly to this group, it can be related to a marked peninsular effect. A parsimony analysis highlights the importance of the oriental part of the Iberian Peninsula as a biogeographical corridor for these species.

Key words: Zygaena, peninsular effect, latitude, longitude, distance from Pyrenees, Mediterranean character, Iberian Peninsula.

* Departamento de Biología (Zoología). Universidad Autónoma de Madrid. Cantoblanco. 28049 Madrid. jose.martin.cano@uam.es 


\section{Introducción}

El efecto península deriva de la teoría del equilibrio dinámico de las islas, propuesta por McArthur \& Wilson $(1963,1967)$ dentro de la hipótesis biogeográficas basadas en las distribuciones por dispersión. Este efecto consiste en la disminución de la riqueza de especies desde el istmo hasta la zona más alejada del continente debido a una mayor tasa de extinción y a una menor tasa de inmigración desde el continente (Real, 1991). Este modelo parece ajustarse bien en algunos grupos taxonómicos y en algunas penínsulas Seib (1980), Means \& Simberloff (1987), pero en otros casos no es así y se ha cuestionado su validez, achacándolo a un artefacto producido por otros factores físicos y climáticos (Busack \& Hedges, 1984).

En las mariposas de la Península Ibérica hay un claro gradiente en la riqueza de especies desde los Pirineos hasta las zonas más alejadas. Los factores geográficos, como son la latitud y de forma más significativa, la longitud y la distancia a los Pirineos aparecen como los factores clave para explicar la abundancia de especies en las distintas áreas ibéricas, y por lo tanto la distribución de mariposas parece ajustarse al modelo peninsular (Martín \& Gurrea, 1990). La existencia de este modelo peninsular en las mariposas ibéricas fue confirmada por Gutiérrez García (1996) basándose en datos de origen muy diferente. Estos datos están basados en las listas de las especies de mariposas en 17 localidades de la Península Ibérica donde había estudios faunísticos de este grupo de lepidópteros.

Una de las causas de que el efecto península esté bastante marcado en las mariposas, se atribuye a un alto grado de homogeneidad en el origen de la fauna de estos insectos. La mayoría de las especies de mariposas parece que han llegado a la Península Ibérica por la vía euroasiática, a través de los Pirineos, y supuestamente hay pocas especies de origen africano (Martín \& Gurrea, 1990). Precisamente Brown (1987) no encuentra efecto península en las mariposas de Baja California, pero como puede deducirse de sus gráficas, esto puede deberse al doble origen de la fauna de mariposas de esa península mexicana. Por un lado hay mariposas norteñas, cuya abundancia va disminuyendo hacia el sur, y por otro lado hay mariposas de origen meridional, que son abundantes en el sur y se van rarificando hacia el norte, dando finalmente una densidad relativamente homogénea al conjunto de especies.

En otro grupo taxonómico de la Península Ibérica, como es la herpetofauna, los gradientes encontrados, tanto en anfibios como en reptiles, son principalmente latitudinales, y se han atribuido al gradiente climático (Schall \& Pianka, 1977). En los coleópteros escarabeidos coprófagos tampoco se ha encontrado un gradiente que indique la generalización de este modelo (Martín Piera et al., 1992) e igual sucede con la riqueza de plantas vasculares (Lobo et al., 2001). En el caso de las especies endémicas de plantas Monocotiledóneas, Colémbolos, Curculiónidos, Lepidópteros y Anfibios y Reptiles, la máxima densidad de microendemismos sí parece darse en las zonas más cercanas a los Pirineos (Martín et al., 2000; GarcíaBarros et al., 2002).

La familia Zygaenidae es un grupo bastante peculiar de lepidópteros. Desde un punto de vista sistemático está incluida dentro de las familias denominadas vulgarmente "polillas" o "heteróceros", esto es lepidópteros generalmente de hábitos nocturnos y colores apagados. Sin embargo muchas de las especies de esta familia son más bien crepusculares o claramente diurnas y de colores vistosos.

La familia Zygaenidae tiene unas 1200 spp. a nivel mundial, de las cuales unas 60 se encuentran en la región Paleártica occidental. Según Vives (1994) en la Península Ibérica la familia está representada por 38 especies incluidas en los géneros Aglope, Theresimima, Rhagades, Adscita y Zygaena. Este último género es el que cuenta con mayor número de especies en la Península Ibérica, en concreto 22, y son las zigenas sensu stricto.

Estas especies del género Zygaena son relativamente uniformes, tienen un tamaño medio, vuelan de día y presentan una coloración viva y llamativa, en la que predominan los colores rojos, blancos y negros. Estos colores se interpretan como aposemáticos y miméticos, y parece que forman grupos sinaposemáticos, ya que unas especies están protegidas porque almacenan productos tóxicos, principalmente derivados del ácido cianídrico, y otras especies parecen imitarlas (Masó et al., 1985; Naumann et al., 1999).

El género Zygaena se subdivide en tres subgéneros: Mesembrynus, Agrumenia y Zygaena. El primero tiene tres especies en la Península Ibérica, el segundo cuatro, y el tercero cuenta con las 15 restantes (Tabla 1). La mayoría de las especies tienen una distribución bastante amplia en Europa, y muchas de ellas están también presentes en Asia. Sólo hay una especie considerada como endemismo ibérico Zygaena (Zygaena) ignifera Korb (1897), cuyas poblaciones se encuentran en tres núcleos: en Teruel, Cuenca y Guadalajara, en Tarragona, y en Jaén y Granada. 
Tabla 1.- Relación de especies de zigenas de la Península Ibérica y número de cuadrículas de $1^{\circ} \mathrm{x} 1^{\circ}$ en las que está presente cada una de ellas.

Table 1.- List of Iberian burnets and the number of $1^{\circ} \mathrm{x} 1^{\circ}$ squares in which each one is present.

\begin{tabular}{lc}
\hline Zygaena Fabricius, 1775 & $\mathbf{n}^{\mathbf{0}}$ de cuadrículas / no. squares \\
\hline Zygaena (Mesembrinus) contaminei Boisduval, 1834 & 9 \\
Zygaena (Mesembrinus) sarpedon (Hübner, 1790) & 70 \\
Zygaena (Mesembrinus) purpuralis (Brünnich, 1763) & 4 \\
Zygaena (Agrumenia) carniolica (Scopoli, 1763) & 8 \\
Zygaena (Agrumenia) occitanica (Villers, 1789) & 39 \\
Zygaena (Agrumenia) hilaris Ochsenheimer, 1808 & 46 \\
Zygaena (Agrumenia) fausta (Linnaeus, 1767) & 55 \\
Zygaena (Zygaena) rhadamanthus (Esper, 1793) & 64 \\
Zygaena (Zygaena) nevadensis Rambur, 1858 & 26 \\
Zygaena (Zygaena) romeo Duponchel, 1835 & 3 \\
Zygaena (Zygaena) osteodensis Reiss, 1921 & 6 \\
Zygaena (Zygaena) exulans (Hochenwarth, 1792) & 5 \\
Zygaena (Zygaena) anthyllidis Boisduval, 1828 & 3 \\
Zygaena (Zygaena) loti (Denis \& Schiffermüller, 1775) & 33 \\
Zygaena (Zygaena) ignifera Korb, 1897 & 7 \\
Zygaena (Zygaena) lavandulae (Esper, 1783) & 47 \\
Zygaena (Zygaena) viciae (Denis \& Schiffermüller, 1775) & 7 \\
Zygaena (Zygaena) ephialtes (Linnaeus, 1767) & 6 \\
Zygaena (Zygaena) transalpina (Esper, 1780) & 20 \\
Zygaena (Zygaena) filipendulae (Linnaeus, 1758) & 47 \\
Zygaena (Zygaena) trifolii (Esper, 1783) & 81 \\
Zygaena (Zygaena) lonicerae (Scheven, 1777) & 28 \\
\hline Media / Average & $\mathbf{2 7 , 9 1}$ \\
\hline
\end{tabular}

Por lo tanto, a pesar de estar relativamente alejadas desde el punto vista sistemático de las mariposas (superfamilias Hesperioidea y Papilionoidea), comparten varias características importantes. Así zigenas y mariposas son de tamaño medio o grande, buenas voladoras, de hábitos diurnos y colores llamativos.

Dadas estas características, las mariposas y zigenas se encuentran entre los grupos de insectos más fáciles de observar en la naturaleza. Y también, y por esto mismo, han sido y siguen siendo uno de los materiales predilectos en las colecciones entomológicas. Todo ello ha contribuido a que se cuenten entre los grupos de insectos ibéricos mejor conocidos al menos desde el punto de vista sistemático y faunístico, y ello permite abordar con ciertas garantías otro tipo de estudios como los biogeográficos.

El objetivo de este trabajo es analizar la distribución de las zigenas de la Península Ibérica estableciendo los factores que tienen mayor poder explicativo, y comparar los resultados obtenidos con los de otros grupos taxonómicos, especialmente con las mariposas.

\section{Método}

Como método para el estudio de la distribución de las zigenas ibéricas se ha utilizado el análisis de densidad de especies en cuadrículas de igual superficie, método ampliamente utilizado. La unidad de la retícula empleada ha sido el grado geográfico, y en total la Península Ibérica tiene 81 cuadrículas de $1^{\circ}$ de latitud por $1^{\circ}$ de longitud, Figura 1. Con esta retícula todas las cuadrículas tienen aproximadamente la misma superficie, excepto las de los límites peninsulares.

La información sobre la distribución de las especies se ha tomado del libro de FernándezRubio (1990a). Una rejilla de $1^{\circ} \times 1^{\circ}$ se ha sobrepuesto sobre los mapas y se ha obtenido la distribución de las especies. Los datos se han tomado en forma de presencia (1), o ausencia (0) de cada especie en cada cuadrícula.

En la nomenclatura de las especies de zigenas se ha seguido el catálogo de Vives (1994). Para el análisis comparado con las mariposas, y sólo a estos efectos, se ha considerado la subfamilia 
A

\begin{tabular}{|l|l|l|l|l|l|l|l|l|l|l|l|l|l|}
\hline 0 & 0 & 0 & 0 & 1 & 2 & 1 & 1 & 1 & & & & & \\
\hline 0 & 0 & 0 & 1 & 2 & 2 & 1 & 1 & 2 & 3 & 3 & 3 & 2 & 1 \\
\hline & 1 & 1 & 1 & 1 & 1 & 1 & 1 & 1 & 1 & 1 & 1 & 1 & 1 \\
\hline & 1 & 1 & 1 & 1 & 2 & 1 & 1 & 1 & 1 & 1 & & & \\
\hline 0 & 1 & 1 & 1 & 1 & 1 & 1 & 1 & 1 & 1 & & & & \\
\hline 0 & 1 & 1 & 1 & 1 & 1 & 1 & 1 & 1 & 1 & 1 & & & \\
\hline & 1 & 1 & 1 & 1 & 1 & 1 & 1 & 1 & 1 & & & & \\
\hline & & 1 & 1 & 1 & 1 & 1 & & & & & & \\
\hline
\end{tabular}

C

\begin{tabular}{|c|c|c|c|c|c|c|c|c|c|c|c|}
\hline 11 & 1 & 3 & -6 & 7 & 5 & 6 & 6 & & & & \\
\hline $1 \% 1$ & 1 & 4 & 7 & 9 & 8 & 9 & 11 & 12 & 11 & 1412 & c) 6 \\
\hline 1 & 2 & 3 & 2 & 3 & 6 & 6 & 6 & 7 & 7 & 109 & 5 \\
\hline 1 & 2 & 3 & 3 & 5 & 6 & 8 & 9 & 8 & 8 & & \\
\hline $2 / 2$ & 2 & 3 & 4 & 3 & 3 & 7 & 9 & 5 & & & \\
\hline $2 \zeta_{2}$ & 2 & 2 & 2 & 2 & 3 & 5 & 4 & 4 & 34 & & \\
\hline 2 & 3 & 3 & 3 & 3 & 6 & 6 & 4 & $\frac{1}{4}$ & & & \\
\hline & & & & 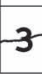 & 3 & 3 & & & & & \\
\hline
\end{tabular}

B

\begin{tabular}{|l|l|l|l|l|l|l|l|l|l|l|l|l|l|}
\hline 0 & 0 & 0 & 0 & 1 & 1 & 1 & 1 & 1 & & & & \\
\hline 0 & 0 & 0 & 0 & 2 & 2 & 3 & 3 & 3 & 4 & 4 & 4 & 4 & 3 \\
\hline & 0 & 0 & 0 & 0 & 2 & 3 & 3 & 3 & 3 & 3 & 3 & 3 & 3 \\
\hline 0 & 0 & 0 & 0 & 1 & 2 & 3 & 4 & 3 & 3 & & & \\
\hline 0 & 1 & 1 & 1 & 1 & 1 & 1 & 1 & 1 & 1 & & & & \\
\hline 0 & 1 & 1 & 1 & 1 & 1 & 1 & 1 & 1 & 1 & 1 & & & \\
\hline & 1 & 1 & 1 & 1 & 1 & 1 & 1 & 1 & 1 & & & & \\
\hline & & & 1 & 1 & 1 & 1 & 1 & & & & & & \\
\hline
\end{tabular}

D

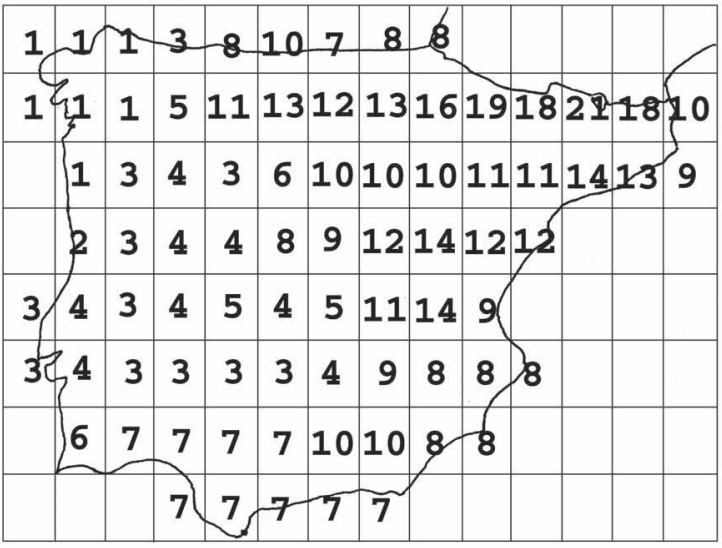

Fig. 1.- Mapas de la densidad de especies de zigenas en la Península Ibérica. Mapa 1a: subgénero Mesembrinus. Mapa 1b: subgénero Agrumenia. Mapa 1c: Subgénero Zygaena. Mapa 1d: género Zygaena.

Fig. 1.- Map with burnet density in the Iberian Peninsula. Map 1a: subgenus Mesembrinus. Map 1b: subgenus Agrumenia. Map 1c: Subgenus Zygaena. Map 1d: genus Zygaena.

Satyrinae con rango de familia e independiente de la familia Nymphalidae.

La densidad de especies se ha contrastado con los siguientes factores geográficos, físicos y climáticos de cada una de las cuadrículas: latitud (Latd), longitud (Long), distancia a Pirineos (DisP), altitud máxima (Almx), altitud mínima (Almn), diferencia de altitud (Aldf) (rango altitudinal), tipo de suelo (Suel), precipitación anual (Prpa) e irregularidad climática (Ircl).

La latitud, la longitud y la distancia a Pirineos se han medido en grados geográficos. En la distancia a Pirineos se ha dado valor 1 a las cuadrículas en las que se encuentra la cordillera pirenaica. En las cuadrículas situadas diagonalmente como valor de la distancia se ha tomado la hipotenusa del triángulo. La latitud y la longitud geográficas son las magnitudes que se utilizan normalmente para situar cualquier localidad geográfica. La distancia al istmo, en este caso a Pirineos, se puede considerar una variable importante en el contexto peninsular, y muy apropiada para valorar el posible efecto península.

La altitud máxima y la altitud mínima miden respectivamente la cota de máxima altitud y de 
Tabla 2.- Datos estadísticos básicos de la densidad de especies de zigenas y de cada uno de los subgéneros en las cuadrículas ibéricas

Table 2.- Basic statistical data of burnet species density and of each of its subgenera in the Iberian squares.

\begin{tabular}{|c|c|c|c|c|}
\hline & Mesembrinus & Agrumenia & subg. Zygaena & Zygaena \\
\hline Número de especies / Species number & 3 & 4 & 15 & 22 \\
\hline Densidad media / Average density & 1,02 & 1,83 & 4,73 & 7,58 \\
\hline Desviación típica / Standard deviation & 0,59 & 1,45 & 3,04 & 4,58 \\
\hline Densidad máxima / Maximum density & 3 & 4 & 14 & 21 \\
\hline Densidad minima / Minimum density & 0 & 0 & 1 & 1 \\
\hline Coeficiente de dispersión / Coefficient of variation & 0,34 & 1,13 & 1,93 & 2,74 \\
\hline
\end{tabular}

menor altitud dentro de cada cuadrícula. Se ha tomado de las hojas 1:200.000 del Servicio Geográfico del Ejercito, y se ha medido en metros. La diferencia de altitud, o rango altitudinal es la diferencia entre la altitud máxima y la altitud mínima de cada cuadrícula. Representa una medida más precisa que la altitud máxima del número niveles altitudinales y por lo tanto de pisos vegetación que puede contener una cuadrícula.

El tipo de suelo se ha empleado como un indicador indirecto de la diversidad vegetal y de hábitats de cada cuadrícula. Dado que las zigenas, como la mayoría de las especies de lepidópteros son fitófagas, una mayor diversidad vegetal podría traducirse en una mayor diversidad de estas especies. En el caso de la zigenas este factor puede ser especialmente relevante, dado que bastantes especies se alimentan en la fase de larva de especies de plantas de preferencias calcícolas. A efectos de cuantificar esta variable se han considerado tres tipos de suelo: silíceo, arcilloso y calizo, y se han dado respectivamente valores $1,1,5$ y 2 . A cada cuadrícula se ha dado un valor que corresponde a la suma de los valores de los diferentes tipos de suelo presentes en ella. Por tanto el valor varía entre 1 y 4,5. El valor máximo corresponde a una cuadrícula con los tres tipos diferentes de suelos aquí considerados. Los tipos de suelo de cada cuadrícula se han tomado de Solé Sabaris (1952).

Los valores de la precipitación media anual se han tomado de Font (1983), y a cada cuadrícula se le ha dado el valor medio.

La irregularidad climática se ha tomado para medir el grado de "mediterraneidad" de cada cuadrícula. Según Montero de Burgos \& González Rebollar (1974) es el factor climático que mejor expresa la carácter mediterráneo de una región. Numéricamente es el coeficiente de variación (desviación típica partida por la media) de la precipita- ción media anual, y mide por tanto la irregularidad de las precipitaciones a lo largo de los años. Los valores se han tomado de estos autores, dando a cada cuadrícula un valor medio.

La precipitación anual y la irregularidad climática cuantifican la disponibilidad de un recurso básico como es el agua para la vegetación, y por lo tanto puede ser un factor importante para la abundancia de especies fitófagas.

El análisis matemático de los resultados se ha realizado mediante la regresión lineal múltiple por pasos sucesivos, empleando como variable dependiente la densidad de especies de zigenas en cada cuadrícula y como variables independientes los nueve factores antes indicados. Los análisis se han efectuado tanto para la densidad de especies del género Zygaena como para cada uno de los tres subgéneros.

Con objeto de estudiar el posible agrupamiento de cuadrículas y de especies de zigenas se han realizado dos análisis de aglomeración. Uno mediante índice de Jaccard, y el otro mediante un análisis de parsimonia.

Los análisis de correlación, regresión y los de aglomeración con el índice de Jaccard se han efectuado con el paquete de programas estadísticos SPSS (SPSS Inc., 2001). El análisis de parsimonia se ha llevado acabo con el programa MIX dentro de paquete de programas PHYLIP (Felsenstein, 1995). El cladrograma de consenso ha sido calculado con el programa CONSENSE, también dentro del paquete de programas PHYLYP.

\section{Resultados}

En los mapas de la Figura 1 se proporciona la densidad de especies en cada cuadrícula de $1^{\circ} \times 1^{\circ}$ para los tres subgéneros y también para el conjunto de las 
Tabla 3.- Valores de los coeficientes de correlación simple entre la densidad de zigenas y los factores considerados.

Table 3.- Values of the correlation coefficient between burnet density and the considered factors.

$$
* \mathrm{p}<0,05 ; * * \mathrm{p}<0,01 ; * * * \mathrm{p}<0,001 ; \text { n.s. no significativo / not significant }
$$

\begin{tabular}{|c|c|c|c|c|c|c|c|c|c|c|c|c|}
\hline & Mesem. & Agrum. & sg. Zyg . & g. Zyg. & Latd & Long & DisP & Almx & Almn & Aldf & Suel & Prpa \\
\hline subgénero Agrumenia & $\begin{array}{r}0,458 \\
* * *\end{array}$ & & & & & & & & & & & \\
\hline subgénero Zygaena & $\begin{array}{r}0,706 \\
* \star *\end{array}$ & $\begin{array}{r}0,682 \\
* \star *\end{array}$ & & & & & & & & & & \\
\hline género Zygaena & $\begin{array}{r}0,742 \\
* * *\end{array}$ & $\begin{array}{r}0,827 \\
* \star *\end{array}$ & $\begin{array}{r}0,970 \\
* * *\end{array}$ & & & & & & & & & \\
\hline Latitud & 0,072 & $-0,231$ & 0,334 & 0,158 & & & & & & & & \\
\hline Longitude & *** & *** & *** & *** & ns & & & & & & & \\
\hline Distancia a Pirineos & $-0,550$ & $-0,489$ & $-0,836$ & $-0,784$ & $-0,504$ & 0,847 & & & & & & \\
\hline Distance from Pyrenees & *** & *** & $* * *$ & *** & $* * *$ & *** & & & & & & \\
\hline Altitud máxima & 0,577 & 0,338 & 0,603 & $-0,780$ & 0,213 & $-0,375$ & $-0,495$ & & & & & \\
\hline Maximun altitude & *** & ** & $* * *$ & $* * *$ & ns & $* * *$ & $* * *$ & & & & & \\
\hline Altitud mínima & 0,368 & 0,175 & 0,381 & 0,356 & 0,094 & $-0,175$ & $-0,371$ & 0,405 & & & & \\
\hline Minimum altitude & *** & ns & $* * *$ & $* * *$ & ns & $\mathrm{ns}$ & $* * *$ & *** & & & & \\
\hline Precipitación anual & $-0,176$ & $-0,463$ & $-0,162$ & $-0,276$ & 0,543 & 0,395 & 0,117 & 0,113 & $-0,263$ & 0,223 & $-0,434$ & \\
\hline Annual precipitation & ns & *** & ns & * & *** & *** & ns & ns & * & * & *** & \\
\hline Irregularidad climática & $-0,022$ & 0,215 & $-0,286$ & $-0,124$ & $-0,848$ & $-0,041$ & 0,361 & $-0,193$ & $-0,016$ & $-0,204$ & 0,335 & $-0,542$ \\
\hline Climatic irregularity & ns & ns & ** & ns & $* * *$ & ns & $* * *$ & ns & ns & ns & ** & \\
\hline
\end{tabular}

zigenas. En la Tabla 2 están los datos estadísticos básicos de la densidad de especies por cuadrícula, como son la media, desviación estándar, máximo y mínimo del número de especies presentes en las cuadrículas. La densidad media de especies por cuadrícula es de 7,58 especies. El máximo número de especies tanto para el género como para cada uno de los subgéneros se da en la región pirenaica, y los valores mínimos se dan en las cuadrículas más occidentales de Galicia y Portugal y también en Andalucía occidental. El valor máximo, 21 especies, se da precisamente en la región pirenaica y en esa cuadrícula están representadas casi la totalidad de las especies, solamente falta el endemismo ibérico $Z$. ignifera.

De las 22 especies de zigenas, la media de la amplitud de la distribución es de 27,91 cuadrículas (Tabla 1), y abarca desde especies de distribución restringida como $Z$. anthyllidis y $Z$. romeo que solamente se encuentran en 3 cuadrículas, hasta especies como Z. trifolii que está presente en las 81 cuadrículas peninsulares. Las siguientes en amplitud de distribución a $Z$. trifolii son $Z$. sarpedon presente en 70 cuadrículas y $Z$. rhadamanthus en 64 . Hay un cierto salto en el número de cuadrículas ocupadas por cada especie. Hay un grupo de 10 especies que ocupan de 3 a 9 cuadrículas, y las 12 restantes pasan a ocupar más de 20 cuadrículas.
En la Tabla 3 se indican los valores y los niveles de significación de los coeficientes de correlación simples entre los grupos taxonómicos analizados y los factores considerados, y de todos ellos entre sí.

Las características geográficas, de suelo y climatológicas de la Península Ibérica hacen que los valores de los coeficientes de correlación de los nueve factores entre sí sean en bastantes ocasiones significativos, y en algunos casos con valores muy altos. En primer lugar el coeficiente de correlación entre la altitud máxima y la diferencia de altitud o rango altitudinal es tan alto que en la práctica son la misma variable, y es difícil distinguir una de otra. Igualmente, el coeficiente de correlación entre la distancia a Pirineos y la longitud, y también con la latitud, aunque con un valor algo menor, es debido a la forma aproximadamente pentagonal de la Península, y a la forma y posición del istmo. Se han mantenido estas dos variables, distancia a Pirineos y longitud, a pesar del alto valor de su coeficiente de correlación por su distinto significado. Las dos son dos sistemas de referencia geográfica arbitrarios, pero mientras la longitud geográfica es de utilización universal, la distancia a Pirineos sólo tiene significado en el contexto geográfico ibérico, pero aquí puede ser muy útil para valorar la hipótesis del efecto península. 
Tabla 4.- Variables introducidas y valores de los coeficientes de correlación lineal múltiple por pasos entre la densidad de zigenas, y de los tres subgéneros con las variables consideradas. Ecuación del hiperplano de regresión para la densidad de especies de las zigenas ibéricas.

Table 4.- Variables used and the values of the stepwise multiple correlation analysis between burnets and the tree subgenera with different variables. Equation of hiperplane regression for species density of Iberian burnets

Latd: Latitud / Latitude. Long: Longitud / Longitude. DisP: Distancia a Pirineos / Distance from Pyrenees. Almx: Altitud máxima / Maximum altitude. Almn: Altitud mínima / Minimum altitude. Aldf: Diferencia de altitud / Altitude difference. Suel: Tipo de suelo / Soil type. Prpa: Precipitación anual / Annual precipitation. Irrcl: Irregularidad climática / Climatic irregularity.

En todos los casos $\mathrm{p}<0,001 /$ In all cases $\mathrm{p}<0,001$

subg. Mesembrinus

\begin{tabular}{l|rrrr}
\hline Variable introducida / Introduced variable & Almx & Suel & Suel \\
valor de $r$ acumulado / cumulative $r$ coefficient & 0,58 & 0,66 & 0,71 \\
subg. Agrumenia & & & \\
\hline Variable introducida / Introduced variable & Long & Suel & DisP & Ircl \\
valor de $r$ acumulado / cumulative $r$ coefficient & 0,71 & 0,83 & 0,84 & 0,85 \\
subg. Zygaena & & & \\
\hline Variable introducida / Introduced variable & DisP & Suel & Almx \\
valor de $r$ acumulado / cumulative $r$ coefficient & 0,84 & 0,87 & 0,89 \\
g. Zygaena & & & & \\
\hline Variable introducida / Introduced variable & Long & Almx & Suel & Ircl \\
valor de $r$ acumulado / cumulative $r$ coefficient & 0,78 & 0,84 & 0,88 & 0,90
\end{tabular}

Ecuación del hiperplano de regresión
Equation of hiperplane regression
$\mathrm{n}^{0} \operatorname{spp}$ Zygaena $=10,578-(0,800$ Long $)+\left(1,344 \times 10^{-3} \mathrm{Almx}\right)+(1,429$ Suel $)-(0,219$ Irrcl $)$

También tiene un valor alto el coeficiente de correlación entre la latitud y la irregularidad climática, el de la correlación entre altitud máxima y la distancia a Pirineos, y el de la correlación entre el tipo de suelo y la precipitación anual, que son debidos a la asimetría constitucional de la Península. En este sentido las mayores precipitaciones se dan en el norte y en el oeste peninsular, dividiendo la Península en lo que tradicionalmente se ha llamado la Iberia húmeda o atlántica y la Iberia seca o mediterránea. La mediterraneidad sin embargo tiene un componente más latitudinal. Desde el punto de vista del sustrato también hay una división de la Península Ibérica en dos mitades, la Iberia ácida o silícea y la Iberia caliza, y por ello el tipo de suelo está significativamente correlacionado con la latitud, con la longitud, con la precipitación anual y con la irregularidad climática. Esta complejidad estructural de la Península es un factor fundamental a la hora de interpretar los resultados y que además dificulta y enmascara las posible interpretaciones.

Los valores de los coeficientes de correlación entre los factores considerados y la densidad de especies de zigenas son altamente significativos $(p<0,001)$ en el caso de la longitud, distancia a Pirineos, altitud máxima y tipo de suelo, con valores negativos para la distancia a Pirineos y la longitud, y positivos para la altitud máxima y el tipo de suelo. El valor del coeficiente de regresión es significativo $(p<0,05)$ para la precipitación media anual, con valor positivo. Para la latitud y la irregularidad climática los valores de los coeficientes no son significativos.

La Tabla 4 recoge los resultados del análisis de regresión lineal por pasos. Para todo el género, la primera variable introducida es la longitud, dado que es la que tiene el mayor valor del coeficiente de regresión simple. En el siguiente paso se introduce la altitud máxima, a continuación el tipo de 
Tabla 5.- Valores de los coeficientes de correlación simple entre la densidad de zigenas, las familias y la totalidad de las mariposas ibéricas.

Table 5.- Values of the correlation coefficient between burnet density, and the different butterfly families, as well as their total.

$$
* \mathrm{p}<0,05 ; * * \mathrm{p}<0,01 ; * * * \mathrm{p}<0,001 ; \text { n.s. no significativo / not significant }
$$

\begin{tabular}{|c|c|c|c|c|c|c|c|c|}
\hline & Zygaena & Mariposas & Hesperioidea & Papilionidae & Pieridae & Anthocharinae & Lycaenidae & Nymphalidae \\
\hline Mariposas / Butterflies & $0,81^{\star * *}$ & & & & & & & \\
\hline Hesperioidea & $0,62 * * *$ & $0,77^{* * *}$ & & & & & & \\
\hline Papilionidae & $0,80^{* * *}$ & $0,74^{* * *}$ & $0,51^{* * *}$ & & & & & \\
\hline Pieridae & $0,52 * * *$ & $0,37^{* * *}$ & $0,48^{* * *}$ & $0,61^{* * *}$ & & & & \\
\hline Anthocharinae & 0,06 n.s. & $-0,12$ n.s. & 0,20 n.s. & 0,28 n.s. & $0,72 * * *$ & & & \\
\hline Lycaenidae & $0,83^{* * *}$ & $0,97 * * *$ & $0,68^{* * *}$ & $0,75^{* * *}$ & $0,34 * * *$ & $-0,13$ n.s. & & \\
\hline Nymphalidae & $0,54 * * *$ & $0,86^{* * *}$ & $0,50 * * *$ & $0,45 * * *$ & $-0,04$ n.s. & $-0,50 * * *$ & $0,83^{* * *}$ & \\
\hline Satyridae & $0,80^{* * *}$ & $0,95 * * *$ & $0,78^{* \star *}$ & $0,75^{* * *}$ & $0,40 * * *$ & $-0,06$ n.s. & $0,89 * * *$ & $0,75^{* * *}$ \\
\hline
\end{tabular}

suelo, y la ultima variable introducida es la irregularidad climática. Ninguna de las variables introducidas ha sido retirada en los sucesivos pasos. El valor final del coeficiente de correlación múltiple 0,902 es significativo $(p<0,001)$. En esta tabla también se recoge la ecuación del hiperplano de regresión para las zigenas, con los valores de la constante y de los coeficientes de cada una de las variables introducidas.

Para los tres subgéneros también se alcanzan coeficientes significativos y con valores muy altos, aunque alguna de las variables introducidas en los pasos sucesivos es distinta.

En la Tabla 5 se recogen los coeficientes de correlación entre las distribuciones de las mariposas, incluyendo las familias, y algún grupo taxonómico, como los Anthocharinae por sus peculiares distribuciones geográficas.

El análisis de agrupamiento de especies utilizando el índice de Jaccard han formado tres grupos (Figura 2). El primero incluye 12 especies y son las que tienen una distribución amplia, que alcanza al menos las zonas cercanas a las costas mediterráneas. El segundo grupo de 9 especies está formado por las especies cuya distribución esta restringida a la región pirenaica, con algunas especies que pueden encontrarse en zonas del interior, pero no en las zonas cercanas a las costas mediterráneas. Finalmente, entre ambos queda un grupo formado por la única especie endémica, Z. ignifera, que a diferencia de las demás especies de zigenas su distribución no alcanza los Pirineos.

El análisis de parsimonia para el agrupamiento de cuadrículas se ha realizado considerando como grupo externo una cuadrícula ocupada por todas las especies. En el cladograma de consenso hay dos clados muy marcados, que dividen a la Península en dos mitades. Por un lado la mitad oriental, Figura 3, que incluye el valle del Ebro, el Sistema Ibérico, Levante, Cazorla y Segura y Sierra Nevada. Por otro lado está la mitad occidental. Finalmente se juntan estos dos clados y se unen a ellos las cuadrículas pirenaicas.

La comparación entre la densidad de la distribución de especies de zigenas con las mariposas, da un valor del coeficiente de correlación significativo muy alto (Tabla 5), e igual sucede con las distintas familias. Solamente no hay significancia en los valores del coeficiente de correlación con la densidad de Anthocharinae.

Los valores más altos del coeficiente de correlación se dan con la familia Lycaenidae, seguida de la Papilionidae, y los más bajos, dentro de los significativos, con las familias Satyridae y Pieridae. Los Anthocharinos también dan coeficientes no significativos con las mariposas en conjunto, y con las familias Hesperiodea, Papilionidae, Lycaenidae y Satyridae.

\section{Discusión}

La elección de la retícula en grados geográficos y no en retícula UTM, se ha hecho para facilitar su comparación con la distribución de las especies de mariposas que está hecha en esta misma retícula (Martín \& Gurrea, 1990).

La distribución de las zigenas la podemos considerar aceptablemente conocida a esta escala. Los mapas de los que se han tomado los datos son la segunda versión que hace el autor de la distribución de las especies ibéricas de este género, y las zigenas son sin duda uno de los grupos de insectos mejor conocidos, y no hay por ahora datos más pre- 


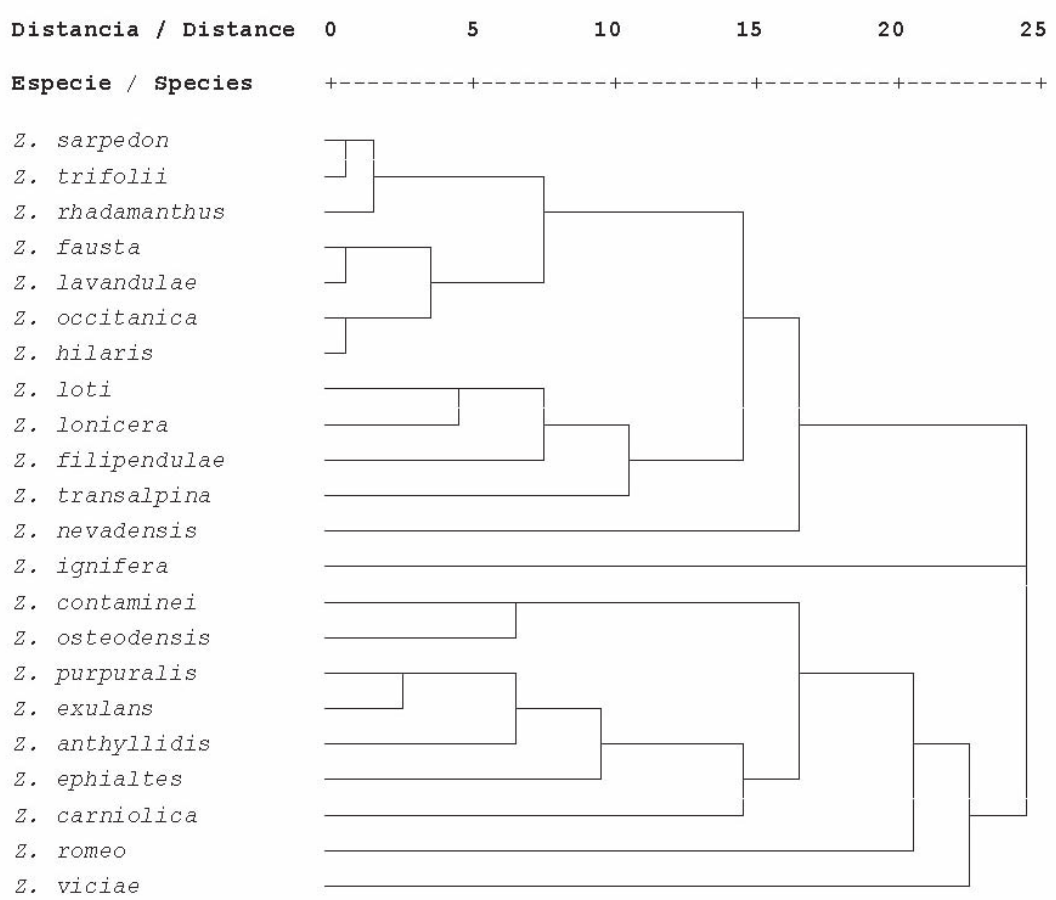

Fig. 2.- Dendrograma de las zigenas ibéricas según las áreas de distribución. Basado en el índice de Jaccard, método de aglomeración UPGMA.

Fig. 2.- Dendrogram of the Iberian burnets using distribution areas based on the Jaccard index and UPGMA.

cisos. Las áreas de distribución de estos mapas son muy similares a las áreas que proporcionan Naumann et al. (1999).

En las dos principales publicaciones españolas sobre lepidópteros, Shilap Revista de Lepidopterología y Butlletí de la Societat Catalana de Lepidopterlogia, aparecen de forma regular notas sobre la distribución de las especies de este género, pero no se ha efectuado ninguna recopilación de las referencias ni revisiones de las principales colecciones entomológicas con vistas a compilar su distribución en mapas más precisos, como cuadrículas UTM de $10 \mathrm{~km}$ y posiblemente estemos lejos de disponer de esta información. En Francia, que han publicado recientemente el atlas de las zigenas francesas, su elaboración desde los primeros prolegómenos ha durado cerca de veinte años, y la recopilación más intensa de datos se prolongó más de diez (Drouet \& Faillie, 1997).

Se ha comparado dentro de lo posible las distribuciones de las zigenas ibéricas de Fernández Rubio (1990a) con algunos de los datos que aparecen en el atlas de las zigenas francesas de Drouet \& Faillie (1997). Este atlas usa principal-

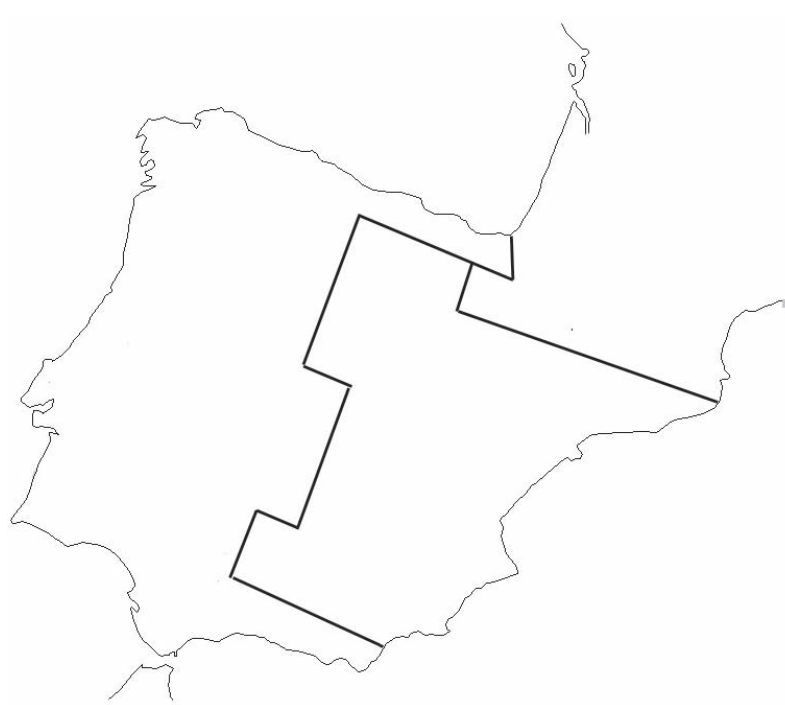

Fig. 3.- Mapa de la Península Ibérica en el que se muestran los dos principales territorios según la agrupación de las cuadrículas efectuada mediante el análisis de parsimonia.

Fig. 3.- Iberian Peninsula map with the two main territories according to the squares grouped using the parsimony analysis. 
mente una retícula UTM de $10 \mathrm{~km}$ de lado, y está basado en una revisión exhaustiva de las publicaciones sobre las zigenas de Francia, y también de las colecciones tanto públicas como privadas. Este atlas de Francia recoge datos muy parciales de la Península Ibérica, sólo los que han aparecido al buscar los datos de Francia, y además en los mapas sólo están representadas las zonas más próximas a los Pirineos. Si comparamos estos pocos datos con los de Fernández Rubio (1990a) no parece haber discrepancias importantes. Sólo en un par de especies ( $Z$. nevadensis y Z. romeo), que según el atlas francés están presentes en algunas cuadrículas que no están señaladas en los mapas de Fernández Rubio (1999a).

El nivel de endemicidad que hay en las zigenas, una especie de un total de 22 que representa aproximadamente el $4,5 \%$ es similar porcentaje de endemicidad que hay en mariposas y noctuidos $(4,2 \%)$, inferior al de otros grupos con menor capacidad de dispersión como curculiónidos $(35,0 \%)$, colémbolos $(32,3 \%)$, anfibios y reptiles $(28,2 \%)$ o monocotiledóneas (18,1\%) (Martín et al., 2000), y algo superior al de los sírfidos (2,4\%) según datos de Marcos García et al. (1998).

Los valores de los coeficientes de correlación de los tres subgéneros entre sí y con la totalidad del género son todos significativos y con valores muy altos, lo cual puede indicar un comportamiento parecido de los tres subgéneros con respecto a su distribución geográfica en la Península Ibérica. Las diferencias en los valores parecen estar relacionadas más con el número de especies que con diferencias en la distribución. El subgénero Mesembrynus con 3 especies y el Agrumenia con 4 están en el límite para un análisis estadístico de este tipo, no obstante se han mantenido los valores porque nos permite comprobar un alto grado de homogeneidad en todo el género.

Las variables elegidas son las mismas que se emplearon para las mariposas (Martín \& Gurrea, 1990). Y estos factores son prácticamente los mismos o muy parecidos a los que han utilizado otros autores para análisis similares en la fauna ibérica, como Schall \& Pianka (1977) para la herpetofauna, Gutiérrez García (1996) para las mariposas, Martín Piera et al. (1992), Lobo \& Martín Piera (2002), y Lobo \& Hortal (2003) para los escarabeidos coprófilos, y también Lobo et al. (2001) para la flora.

Los niveles de significación de las variables, tanto los de las correlaciones simples como los de regresión múltiple son en la mayoría de los casos significativos y con una alta absorción de varianza, lo que les confiere un alto valor como factores para explicar la distribución de este grupo de lepidópteros en la Península Ibérica.

La longitud geográfica, la distancia a Pirineos y la altitud máxima son los factores que absorben la mayor varianza, y también son las primeras variables introducidas en la regresión múltiple por pasos. Desde el punto de vista de la regresión múltiple, las otras variables como el tipo de suelo o la irregularidad climática parecen tener un papel menos relevante en el número de especies de zigenas presentes en un territorio. Y finalmente, la latitud y la precipitación anual no parecen tener influencia en el número de especies de zigenas presentes en la región, aunque esto se deba probablemente a que su posible contribución al total de la varianza absorbida es tomada por otras variables. Estos resultados son parecidos a los obtenidos para las mariposas (Martín \& Gurrea, 1990), y puede ser debido a que las mariposas y las zigenas tienen un patrón similar de distribución en la Península Ibérica.

En el caso de las mariposas, la primera variable absorbida por el análisis multivariante era la distancia a Pirineos, lo que se interpretaba como un "efecto península". En el caso de zigenas la primera variable absorbida es la longitud, sin embargo la distancia a Pirineos es tomada como primera variable para las especies del subgénero Zygaena, lo cual nos permite ver la similitud de ambas variables en el contexto ibérico.

El coeficiente de correlación simple entre la longitud geográfica y la distancia a Pirineos no sólo es significativo, sino que tiene un valor muy alto $(0,847)$ debido a la forma de la Península Ibérica. Las cuadrículas mas alejadas de los Pirineos son las que corresponden a Galicia, Portugal y Andalucía occidental, que son también las más occidentales y por lo tanto las que tienen un valor mayor en la longitud geográfica. Por el contrario las cuadrículas más orientales, como son las de Cataluña, Aragón y Levante están también cerca de Pirineos.

Si analizamos también el papel que la longitud geográfica puede jugar en la distribución de las especies la interpretación más evidente es que representa un gradiente de alejamiento de las áreas de origen de las especies, y por lo tanto en el caso ibérico asimilable a un efecto península. Otras interpretaciones como que este enmascarando un efecto climático o de otro tipo no parecen muy consistentes. Si bien la longitud está correlacionada de forma significativa con la precipitación anual, el valor que tiene, 0,395 , es mayor que el que tiene la precipitación anual con la distribución de la zige- 
nas, $-0,276$ y por lo tanto se puede descartar un efecto debido simplemente a un gradiente en las precipitaciones anuales. La longitud geográfica también está correlacionada con la altitud máxima de cada cuadrícula, debido a que buena parte de las altitudes mayores dentro de las zonas montañosas se concentran más en la zona oriental de la Península Ibérica, como es el caso de los Pirineos, el Sistema Ibérico, o Sierra Nevada. También en este caso el valor del coeficiente de correlación es mayor para la longitud que para la altitud máxima, y por lo tanto no se puede explicar totalmente el valor para la correlación simplemente por influencia de la correlación entre la longitud y la altitud máxima. Insistiendo en este mismo sentido, la altitud máxima es tomada por el programa de regresión múltiple por pasos detrás de la longitud geográfica, y contribuye incrementando la varianza absorbida de forma conjunta, y por ello el papel de la longitud geográfica no puede atribuirse a un simple efecto derivado de su correlación con la altitud máxima de cada cuadrícula.

Hay ciertas diferencias entre los valores encontrados en las zigenas y también en las mariposas de los encontrados por otros autores para los coleópteros escarabeidos coprófagos y para las plantas vasculares. Tanto para los escarabeidos coprófagos (Martín Piera et al., 1992; Lobo \& Martín Piera, 1997; Lobo \& Hortal, 2003) como para las plantas vasculares (Lobo et al., 2001) la variable con el valor del coeficiente de correlación simple más alto, y también la que es tomada en primer lugar en los análisis multivariantes es la altitud o elevación máxima de cada cuadrícula. En segundo lugar aparecen otras variables como el clima para las plantas, o algunos factores más específicos, como es el uso del suelo para los escarabeidos coprófilos. En tercer lugar aparecen otros factores como la diversidad geológica (similar al tipo de suelo). En el caso de los escarabeidos aparece finalmente la latitud. La longitud geográfica y la distancia a Pirineos no dan valores significativos, o no son absorbidas en los análisis multivariantes, y por ello, en estos casos no se ha considerado la existencia de un efecto península.

Tanto las escarabeidos coprófilos como obviamente las plantas vasculares, son grupos muy complejos y heterogéneos en sus orígenes, contando con elementos procedentes de muy distintas áreas geográficas. Lógicamente no puede darse un efecto península en grupos de composición muy diferente, en los cuales la vía peninsular, o sea la entrada desde el continente hacia el istmo y después dispersión por la península es sólo una opción más

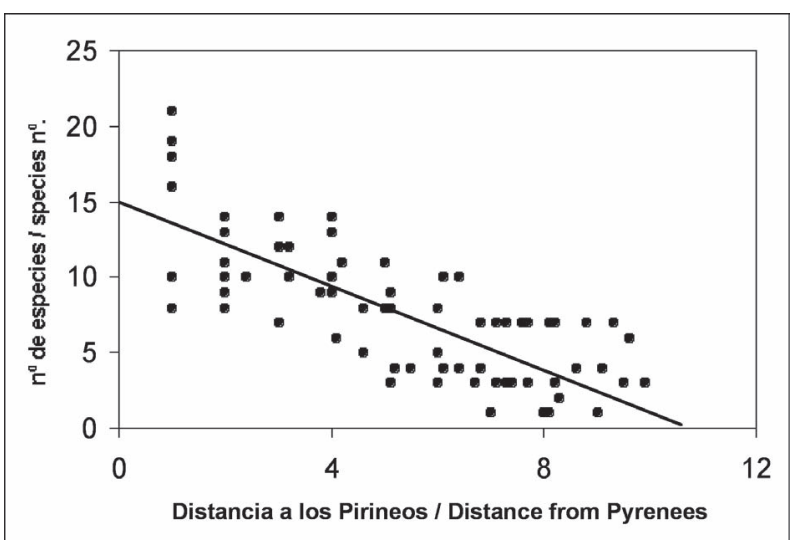

Fig. 4.- Relación entre la densidad de especies de zigenas y distancia a los Pirineos. (Distancia a los Pirineos en grados geográficos).

Fig. 4.- Burnet species density and distance from the Pyrenees relationship. (Distance from de Pyrenees in geographical degrees).

frente a otros grupos de especies que han entrado por la vía africana, o por otros caminos, o incluso en épocas anteriores donde no se podía hablar de una península como tal. Sin embargo en zigenas, al igual que en mariposas, parece que el origen es bastante homogéneo, y una mayoría de especies son de origen europeo lo que ha dado lugar a una distribución que sigue un modelo peninsular, y la Figura 4 es bastante elocuente de este fenómeno para las zigenas.

Comparando la densidad de especies de zigenas con las de las distintas familias de mariposas, los valores más altos se dan con la familia de los Licénidos y los más bajos con las familias de los Piéridos y de los Ninfálidos. Las diferencias de los patrones de distribución entre los Licénidos y los Ninfálidos fueron atribuidas (Martín \& Gurrea, 1990) en base a la mayor capacidad de vuelo en general de los Ninfálidos, familia que cuenta con muchas especies vágiles y de gran tamaño, y por ello parecen depender menos de los factores geográficos y más de los climáticos en sus distribuciones. La zigenas por su tamaño, y posible capacidad de vuelo estarían más cerca de las familias Licénidos, y también de los Hespéridos y los Papiliónidos.

Los valores más bajos con los Piéridos se pueden explicar por el origen mixto de esta familia de mariposas. Dentro de los Piéridos, los Anthocarinos parecen tener un origen africano, y por lo tanto no 
europeo como la mayoría de las mariposas. Precisamente el valor del coeficiente de correlación entre los Antocarinos y las zigenas no es significativo, y se puede atribuir también a la ausencia o por lo menos escasez de elementos de origen africano entre las especies de zigenas. Una buena parte de la zigenas ibérica tienen una amplia distribución en Europa, principalmente en zonas de clima atlántico o continental, y bastantes llegan a Asia, posiblemente el área de origen de las zigenas europeas (Fernández Rubio, 1990b; Nauman et al. 1999).

En este mismo sentido las zigenas tienen un valor del coeficiente de correlación con la irregularidad climática no significativo y negativo aunque muy próximo a cero. En el caso del subgénero Zygaena, el valor del coeficiente de correlación con la irregularidad climática es ligeramente significativo y con una absorción moderada de la varianza.

Además este valor es negativo, lo que refuerza la hipótesis de la predominancia de elementos euro-atlánticos y la ausencia o por lo menos escasez de componentes mediterráneos dentro de las zigenas ibéricas.

El análisis biogeográfico por parsimonia se ha empleado en numerosas ocasiones principalmente para estudios sobre la endemicidad, pero también como sistema para estimar la asociación de especies o territorios Vargas (1991), Posadas (1996), Posadas et al. (1997, 1999). Lo habitual es considerar como grupo externo o estado plesiomorfo la ausencia de las especies (Vargas, 1991; Morrone, 1994; García-Barros et al. 2002), pero en este caso se ha tomado como grupo externo la presencia de especies. Lógicamente podemos pensar como un estado anterior una cuadrícula vacía o la falta de una especie, que llegará con posterioridad a ocupar la cuadrícula, bien sea por vicarianza o por invasión. En el caso de la Península Ibérica podemos pensar que las especies han estado sufriendo expansiones hacia el sur en las épocas glaciares, y contracciones hacia el norte en los periodos interglaciares, y podríamos considerar que las especies en la glaciación anterior han tenido en una distribución algo más amplia, por lo menos hacia el sur. $\mathrm{La}$ distribución actual supuestamente es una contracción de la distribución durante el periodo glaciar, y por lo tanto estaría justificado considerar como estado plesiomorfo una distribución más amplia de estas especies.

Las agrupaciones del territorio peninsular efectuadas por el análisis de parsimonia ponen de relieve el papel que ha jugado la distribución de las montañas ibéricas durante las glaciaciones. La zona oriental queda enlazada por el Sistema Ibérico, que al estar colocado de forma paralela a los meridianos actúa de corredor norte-sur durante los cambios de periodos glaciares a interglaciares y viceversa. Frente a esto, la mitad occidental, que tiene la Cordillera Cantábrica, el Sistema Central, los Montes de Toledo, y las Béticas colocadas de forma transversal a los meridianos dificultando el flujo de especies hacia el norte en los periodos interglaciares y hacia el sur en las épocas glaciares.

Finalmente, se podría hablar también de dos Iberias, la Iberia oriental, con una alta conectividad que facilita el flujo de especies de ciertos grupos taxonómicos en dirección norte-sur, frente a una Iberia occidental, con una baja conectividad nortesur. Esta facilidad diferencial de paso de especies entre la mitad oriental y la mitad occidental puede ser la causa de la riqueza de especies de mariposas y zigenas en la zona oriental y su menor número en la mitad occidental.

\section{AGRADECIMIENTOS}

J.L. González Rebollar proporcionó los datos de la irregularidad climática en Portugal.

\section{Referencias}

Brown, J. W., 1987. The peninsular effect in Baja California: an entomological assessment. Journal of Biogeography, 14: 359-365.

Busack, S. D. \& Hedges, S. B., 1984. Is the peninsular effect a red herring? The American Naturalist, 123, 2: 266-275.

Drouet, E. \& Faillie, L., 1997. Atlas des espèces françaises du genre Zygaena Fabricius. Edition J.-M. Desse. 74 pp.

Felsenstein, J., 1995. PHYLYP (Phylogeny Inference Package) version 3.57 c. University of Washington, Seattle. Disponible en:

http://evolution/genetics.washington.edu/phylyp/software.html http://www.cmbi.kun.nl/bioinf/PHYLIP/main-5.html\#ss5.1

Fernández-Rubio, F., 1990a. Guía de las mariposas diurnas de la Península Ibérica. Zygenas. Pirámide. Madrid. 167 pp.

FERnÁNDEZ-Rubio, F., 1990b. Origen y distribución del género Zygaena Fabricius, 1775 (Lepidoptera) en la Península Ibérica. Boletín de Sanidad Vegetal y Plagas, 16: 455-477.

FonT, I., 1983. Atlas climático de España. Instituto Nacional de Meteorología. Madrid. 43 láms.

García-Barros, E., Gurrea, P., Luciañez, M. J., Martín Cano, J., Munguira, M. L., Moreno, J. C., SAinz, H., SAnZ, M. J. \& SimÓn, J. C., 2002. 
Parsimony analisis of endemicity and its application to animal and plant geographical distributions in the Ibero-Balearic region (western Mediterranean). Journal of Biogeography, 29: 109-124.

GutiÉRrez GARCíA, D., 1996. Variaciones espacio-temporales de los agregados de especies de lepidópteros ropalóceros en los Picos de Europa (Norte de España). Tesis doctoral (inédita). Universidad de Oviedo. 208 pp.

Lobo, J. M., CAstro, I. \& Moreno, J. C., 2001. Spatial and environmental determinants of vascular plant species richness distribution in the Iberian Peninsula and Balearic Islands. Biological Journal of the Linnean Society, 73: 233-253.

Lobo, J. M. \& Hortal, J., 2003. Modelos predictivos: Un atajo para describir la distribución de la diversidad biológica. Ecosistemas, 2003/1: 8 pp. (disponible en URL:

http://www.aeet.org/ecosistemas/031/investigacion3.htm)

Lobo, J. M. \& MArtín Piera, F., 2002. Searching for a Predictive Model for Species Richness of Iberian Dung Beetle Based on Spatial and Environmental Variables. Conservation Biology, 16: 158-173.

Marcos García, M. M., Isidro, P. M., Rojo, S. \& PéreZ BAÑON, C., 1998. Catálogo y distribución geográfica de los sírfidos iberobaleares (Diptera, Syrphidae). I.Syrphinae y Microdontinae. Boletín de la Asociación española de Entomología, 22(3-4): 37-61.

Martín, J. \& Gurrea, P., 1990. The peninsular effect in Iberian butterflies (Lepidoptera: Papilionoidea and Hesperioidea). Journal of Biogeography, 17: 85-96.

Martín, J., García-Barros, E., Gurrea, P., Luciañez, M. J., Munguira, M. L., SAnz, M. J. \& Simón, J. C., 2000. High endemism areas in the Iberian Peninsula. Belgian Journal of Entomology, 2: 47-57.

Martín Piera, F., Veiga, C. M. \& Lobo, J. M., 1992. Ecology and biogeography of dung beetle communities (Coleoptera, Scarabeoidea) in an Iberian mountain range. Journal of Biogeography, 19: 677-691.

Masó, A., Pérez de-Gregorio, J. J. \& Vallhonrat, F., 1985. La vida de les papallones. Ketres. Barcelona. 296 pp.

McArthur, R. H. \& Wilson, E. O., 1963. An equilibrium theory of insular zoogeography. Evolution, 17: 373-387.

McArthur, R. H. \& Wilson, E. O., 1967. The theory of island biogeography. Princeton University Press. Princetown. 203 pp.

Means, D. B. \& Simberloff, D., 1987. The peninsula effect: habitat-correlated species decline in Florida's herpetofauna. Journal of Biogeography. 14: 551-568.
Montero de Burgos, J. L. \& GonzÁlez Rebollar, J. L., 1974. Diagramas Bioclimáticos. ICONA. Madrid. $379 \mathrm{pp}$.

Morrone, J. J., 1994. On the identification of Areas of Endemism. Systematic Biology, 43: 438-441.

Naumann, C. M., Tarmann, G. M. \& Tremewan, W. G., 1999. The Westwern Palaearctic Zygaenidae (Lepidoptera). Apollo Books. Stenstrup. 304 pp.

PosADAS, P., 1996. Distributional patterns of vascular plants in Tierra del Fuego: a study applying parsimony analysis of endemicity (PAE). Biogeographica, 72(4): 161-177.

Posadas, P., Estévez, J. M. \& Morrone, J. J., 1997. Distributional patterns and endemism areas of vascular plants in the Andean subregion. Fontqueria, 48: 1-10.

Posadas, P. \& Miranda-Esquivel, D. R., 1999. El PAE (Parsimony Analysis of Endemicity) como una herramienta en la evaluación de la biodiversidad. Revista Chilena de Historia Natural, 72: 539-546.

REAL, R., 1991. Las tendencias geográficas de la riqueza específica. In: J. M. Vargas, R. Real \& A. Antúnez (eds.). Objetivos y métodos biogeográficos. Aplicaciones en Herpetología. Monografías de Herpetología, 2: 85-94.

SEIB, R. L., 1980. Baja California: a peninsula for rodents but not for reptiles. The American Naturalist, 115: 613-620.

SCHAll, J. J. \& PiAnKA, E. R., 1977. Species densities of Reptiles and Amphibians on the Iberian Peninsula. Doñana, Acta Vertebrata, 4: 27-34.

Solé SABArís, L., 1952. Geografía Física. In: M. Terán (ed.). Geografía de España y Portugal, tomo 1. Montaner y Simón. Barcelona. 498 pp.

SPSS INC., 2001. SPSS para Windows. SPSS Inc. Chicago.

VARGas, J. M., 1991. Escuelas y tendencias en Biogeografía histórica. In: J. M. Vargas, R. Real \& A. Antúnez (eds.). Objetivos y métodos biogeográficos. Aplicaciones en Herpetología. Monografías de Herpetología, 2: 107-136.

VIVES, A., 1994. Catálogo sistemático y sinonímico de los Lepidópteros de la Península Ibérica y Baleares (Insecta: Lepidoptera) (Segunda Parte). Ministerio de Agricultura, Pesca y Alimentación. Madrid. 775 pp. 